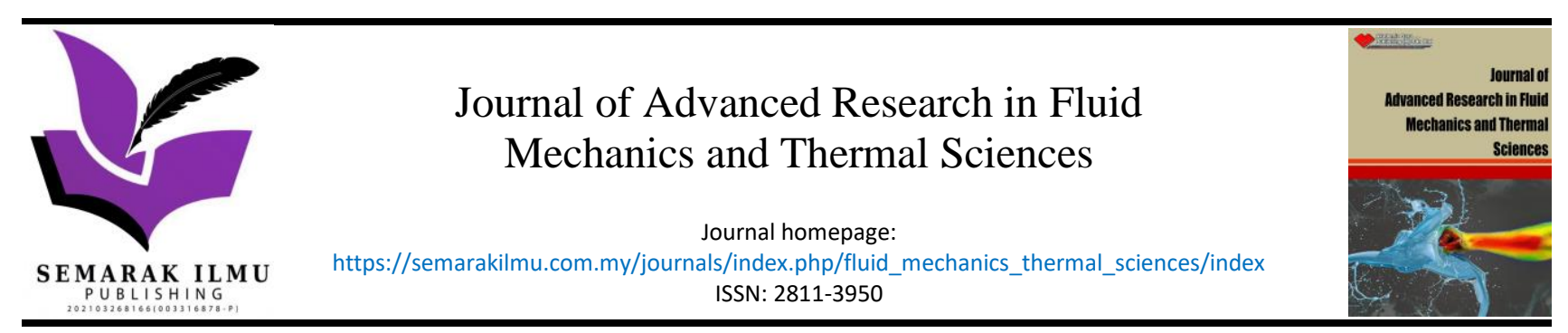

\title{
Enhancing Dynamic Response of Cantilevered Rectangular Prism Using a Splitter Plate as a Passive Turbulence Control in Water Tunnel
}

\author{
La Ode Ahmad Barata ${ }^{1,}{ }^{*}$, Edward Ngii ${ }^{2}$, Takahiro Kiwata ${ }^{3}$, Takaaki Kono ${ }^{3}$ \\ Department of Mechanical Engineering, Halu Oleo University, 93123 Kendari, Indonesia \\ Department of Civil Engineering, Halu Oleo University, Kendari, Indonesia \\ Division of Mechanical Science and Engineering, Kanazawa University, Japan
}

\section{Article history:}

Received 21 July 2021

Received in revised form 25 December 2021

Accepted 28 December 2021

Available online 23 January 2022

\section{Keywords:}

Splitter plate; Rectangular; Dynamic Response; Flow features

\begin{abstract}
The effects of splitter plate as a passive turbulence control on the transverse vibration characteristics of a cantilevered rectangular prism with the different finite length (aspect ratio $L / H$ ) and depth ratios (side ratio $D / H$ of 0.2 and 0.5 ) was investigated in a water tunnel. A splitter plate was installed behind the prism with a small gap and sufficient length to prevent vortex shedding intrusion. It enhances the response amplitude ascended linearly with respect to non-dimensional velocity. In the case of the prisms with critical points, interferences of amplitude signal spectra for the period of displacement vanished, yielding a uniform waveform on the response amplitude. Inserting a splitter plate improves the increment rate of the dynamic response of the test models. A brief numerical calculation was performed to find the quantities of flow features around a plain and rectangular prism with a detached splitter plate. The alteration of flow wake characteristics is found by diminishing unsymmetrical vortices shedding and reduce flow entrainment at the trailing edge. The essential findings of the prism with a detached splitter plate are the base pressure improvement, reducing drag force, and vanishing sharp peaks of wake FFT spectra.
\end{abstract}

\section{Introduction}

The separation shear layer is fixed at the leading edge in the presence of sharp edges on the prismatic structure, such as rectangular and blunt bodies with a flat surface facing the uniform flow. The alternating vortices formation downstream, reattachment point, and shear layer separation at the prismatic structure's trailing edge affects the elastically mounted structure's vibration characteristics. Wake flow characteristics such as the fluid force and Karman vortex shedding are changed significantly in a rectangular prism by the depth ratio. The features of the elastically mounted prismatic structure, such as a rectangular prism subjected to the flow-induced vibration, depending on the damping mechanism and the hydrodynamic shape of the downstream body that is the structural part of a prismatic afterbody shape of the flow separation point [1-2].

\footnotetext{
* Corresponding author.

E-mail address: ahmad.barata@uho.ac.id
}

https://doi.org/10.37934/arfmts.91.2.114 
The effect of cross-section depth on the flow-induced vibration characteristics of the finite length prismatic structure was also investigated by Kiwata et al., [3]. They found that the prisms with slender cross-sections had low onset vibration, high increment rate, and large dynamic response. Nakamura and Hirata [4] plotted the dynamic response characteristics of the rectangular prismatic body with various depth to cross-section height ratios. The rectangular prismatic bodies show a wide range of vibration or galloping types, distinctly different from the elastically mounted circular cylinder defined in the Griffin plot [5]. The dynamic response of the rectangular prisms has a limitation response below the critical depth because of the flow features variations. The peak of base pressure of various depths of the prismatic structure can be found in Nakamura and Matsukawa [6].

The variation of wake structure along spanwise of the prismatic structure results in some studies on the span length effect on the dynamic response and wake structure characteristics such as PalauSalvador et al., Sumner et al., and Wang et al., [7-9]. The dynamic response characteristic of blunt bodies with the span length variations (aspect ratios) influence vibration characteristics for which a particular aspect ratio showed distinctly different characteristics with its counterparts [10]. The flowstructure characteristics also reveal that wake flow characteristics of prismatic structure with below the critical point of the aspect ratios $(\mathrm{L} / \mathrm{H}) \leq 5$ are distinctly different and considerably controlled by the three-dimensionality of vortex structure from the tip end of the prism and the alternating vortex shedding at the downstream of the bluff body. The investigation of flow wake around circular with span length below critical point was also detailed in Gonçalves et al., [11].

Controlling unsteady vortex behaviour in the trailing edge is supposed to diminish strong unsymmetrical vortices resonance due to the separated shear layer interaction. The classical method to control the shear layer interaction has been introduced by Zdravkovich [12]. The alteration of flow wake behaviour is the main objective in inserting a splitter plate as a passive control of unsteady flow behind the prism. Many researchers have focused on numerical and experimental investigations on the attached splitter plate length and gap variation. The presence of a critical gap (a detached splitter plate) and the length variation of the splitter plate is the main consideration in applying for a splitter plate as a passive turbulence control for both rectangular and circular cylinders. The difference of separation points for both cylinders is an essential parameter in the gap and length variation of a splitter plate. A splitter plate changes the flow features, reducing drag, and galloping characteristics for an elastically mounted prism [13 - 17].

The recent extensive studies on the wake stabiliser using a splitter plate have been developed using a flexible splitter plate that enables flap, parallel and multiple, dual, and slotted splitter plates. The flexibility of the flapping splitter plate attached to the elastically mounted circular cylinder showed the alteration vibration mechanism. Increasing the flexibility of the splitter plate tend to change galloping instability to vortex-induced excitation. Contrary to fixed splitter plate, it is uptick galloping instability for rectangular prism and experiencing instability type vibration for the circular and square cylinder [18 - 20]. Assi and Bearman [21] investigated the effect of slotted splitter plates on the vibration characteristic of a circular cylinder. They revealed that the vibration mechanism of a circular cylinder with a splitter plate was not resonant type vibration. Mounting a slotted splitter at the trailing edge of the circular cylinder deflate fluid energy absorption of the vibrated cylinder by decreasing negative damping and pressure differences between splitter plate surfaces.

Hwang and Yang [22]; Barman and Bhattacharyya [23] presented the effect of dual splitter plate (upstream and downstream splitter plate) on flow features around circular and square cylinders. An upstream splitter plate does not affect wake shedding at the trailing edge but reduces drag force. Inserting the upstream and downstream splitter plates reduced drag coefficient but did not increase shedding suppression of downstream wake. 
Parallel splitter plates on downstream cylinders as passive flow separation control have also been demonstrated by Dash, Triantalfyllou, and Alfarado [24]. They showed that double splitter plates are better than a single one for controlling unsteady flow at the trailing edge, advancing pressure recovery and vortex shedding, and suppressing fluctuation of the aerodynamic forces.

However, the effect of a detached splitter plate as passive unsteady flow control in the wake on the vibration response of the cantilevered rectangular prism with the aspect ratio below the critical point has not been explained in detail in the literature. The vibration mechanism of a rectangular prism is distinctly different from that of the square and circular cylinder. It is essential to elucidate the effect of a detached splitter plate on the slender rectangular prism's vibration response and flow features.

This work focused on the instability galloping for the cantilever mounted rectangular of blunt bodies with depth to cross-section height (side ratio, $\mathrm{D} / \mathrm{H}) \leq 0.5$ and various aspect ratios $(\mathrm{L} / \mathrm{H})$. A detached splitter plate for the wake control system at the trailing edge was supposed to enhance the prism's dynamic response to support power generation systems' development from flow-induced vibration in water flow.

\section{Experimental setup}

\subsection{Experimental Apparatus}

The experiments of the dynamic response of cantilevered rectangular prism were conducted in a closed-loop water tunnel facility. Detail of the apparatus used in this study is similar, as in Barata et al., and Mizukami $[10,25]$. The schematic diagram of the test section, and measurement devices, is shown in Figure 1 below. The test section dimension was $400 \times 167 \times 780 \mathrm{~mm}$. The uniform flow velocity $U$ was regulated in the range of $0.74 \mathrm{~m} / \mathrm{s}$ and $2.7 \mathrm{~m} / \mathrm{s}$ by adjusting the pump motor's input frequency and gate opening scale of the control valve. Flow stream velocity was measured by a pitot tube and a digital differential pressure gauge (NAGANO KEIKE, GC50).

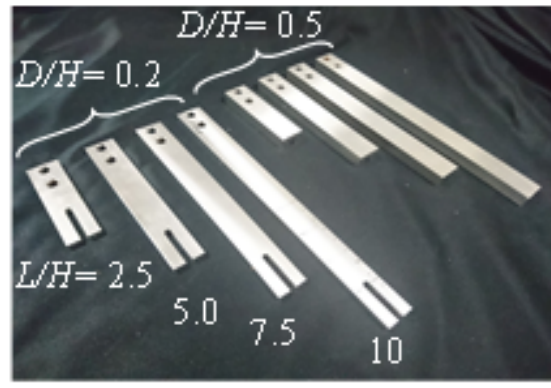

(a)

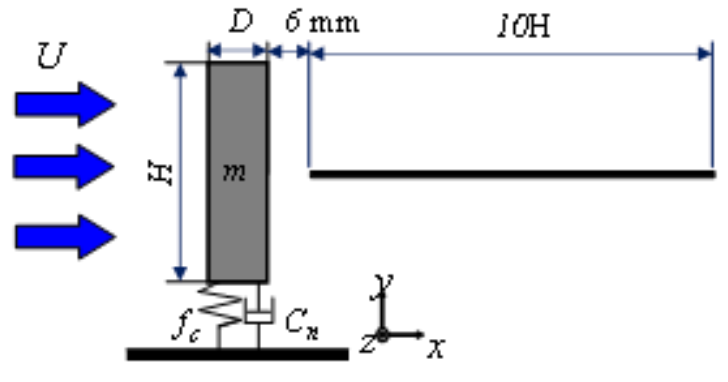

(b)

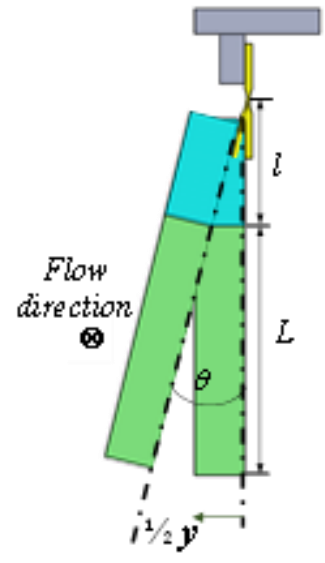

(c)

Fig. 1. (a) Cross-section of the rectangular prisms, (b) Schematic of the FIV test model with a splitter plate (c) FIV translational motion concept

The stationary splitter plate length was $200 \mathrm{~mm}$ or $10 \mathrm{H}$ with a thickness of $1.0 \mathrm{~mm}$. It was installed on the central prism axis behind the prism. The gap between the prism trailing edge and the splitter was around $1 / 4 \mathrm{H}$. According to the references, the splitter plate length and gap are enough to stabilise unsteady vortex shedding in the streamwise direction $[16,26]$. 


\subsection{Test Models and Procedures}

The test models were constructed from a smooth stainless steel surface and mounted elastically using a flexible plate hooked up to the upper wall inside a water tunnel of the test section. The prism displacement was one degree of freedom, i.e., transverse direction. The schematic of the test models and test section is detailed in Figure 1. The rectangular prisms had the constant cross-section height $\mathrm{H}$ of $20 \mathrm{~mm}$ and two different depths $\mathrm{D}$, i.e. 5 and $10 \mathrm{~mm}$. The model's depth with the side ratio (D/H) of 0.5 is transitional galloping type behaviour for an elastically mounted bluff body. This study considers that the test model with a side ratio of 0.2 is a pure instability galloping type. Figure $1(a)$ shows the cross-section models with different side ratios, $D / H$ (D: the downstream dimension of the prism, and $\mathrm{H}$ is cross-section height facing flow stream direction) of the rectangular prisms of 0.2 and 0.5 . The lengths of the prisms test $(\mathrm{L})$ were $50,100,150$, and $200 \mathrm{~mm}$ with the aspect ratios $\mathrm{L} / \mathrm{H}$ of $2.5,5.0,7.5$, and 10 .

Before conducting the flow-induced vibration test, the initial displacement test and frequencycharacteristic $f_{\mathrm{c}}$ of the system were measured by hitting the prism at the air and stationary water flow. Frequency characteristics $f_{c}$ was set in the range of 16 and $39 \mathrm{~Hz}$ by using different plate thicknesses. The reduced-mass damping parameter $\left(C_{n}\right)$ was taken by measuring the decrement rate of structural damping $(\delta)$, which is defined as logarithmic of peak displacement, $p$ at the time $\left(i^{\text {th }}\right)$ over peak displacement $p$ at time $(i+n)^{\text {th }}$ as defined in Figure 2 . These non-dimensional quantities are defined as:

$$
\begin{gathered}
\delta_{i}=\ln \frac{p_{i}}{p_{i+n}} \\
C_{n}=2 \delta \times \frac{\rho_{s}}{\rho_{f}}
\end{gathered}
$$

where $\rho$ is the density of structure $(s)$ and fluid $(f)$. Figure 2 below demonstrates the structural damping for which the decay rate of free vibration tests is defined. Table 1 summarises the physical properties of the test models. The test model's structural damping into the water is less than in the air because of the viscous effect and added mass. The disparity of the test model's frequency characteristic in the water and air is around $2-5 \%$.

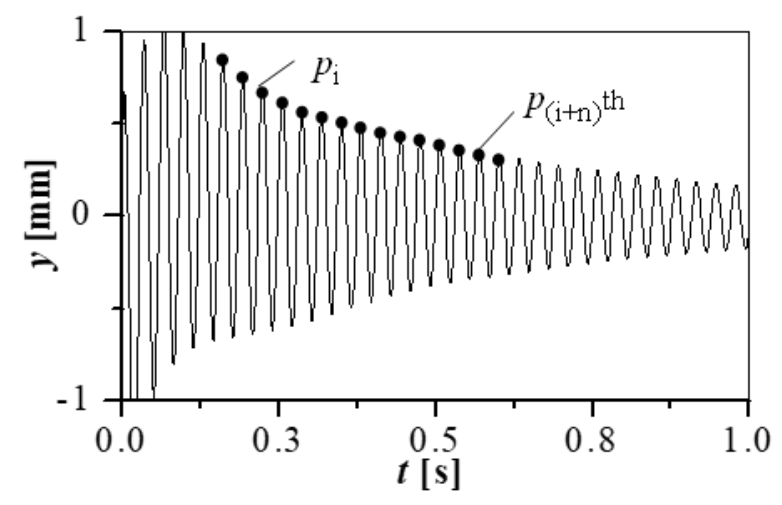

Fig. 2. The decay rate of response amplitude conceptual 


\section{Tabel 1}

Physical properties of the test models

\begin{tabular}{|c|c|c|c|c|c|c|c|c|}
\hline \multicolumn{3}{|l|}{ Properties } & \multicolumn{3}{|c|}{ In the water } & \multicolumn{3}{|c|}{ In the air } \\
\hline $\begin{array}{l}\text { Cross section } \\
\text { Model }\end{array}$ & $D / H$ & Aspect Ratio $(L / H)$ & $f_{c}[\mathrm{~Hz}]$ & $\delta$ & $C n$ & $f_{c}[\mathrm{~Hz}]$ & $\delta$ & $C n$ \\
\hline Plain & 0.20 & 10.0 & 26.49 & 0.0262 & 0.46 & 27.08 & 0.0117 & 152.26 \\
\hline \multirow[t]{7}{*}{ Rectangular } & & 7.5 & 26.36 & 0.0504 & 0.89 & 26.86 & 0.0125 & 162.92 \\
\hline & & 5.0 & 38.70 & 0.0262 & 0.46 & 39.23 & 0.0140 & 181.71 \\
\hline & & 2.5 & 38.45 & 0.0224 & 0.39 & 38.70 & 0.0146 & 190.07 \\
\hline & 0.50 & 10.0 & 16.36 & 0.0314 & 0.55 & 16.97 & 0.0115 & 149.68 \\
\hline & & 7.5 & 16.48 & 0.0296 & 0.52 & 16.97 & 0.0151 & 196.21 \\
\hline & & 5.0 & 24.78 & 0.0263 & 0.46 & 25.63 & 0.0147 & 191.52 \\
\hline & & 2.5 & 26.37 & 0.0225 & 0.40 & 26.86 & 0.0163 & 211.95 \\
\hline
\end{tabular}

An embedded type acceleration sensor (Showa Measuring Instrument, $2302 \mathrm{CW}$ ) in the tip of the test model was used to measure the model's tip displacement. The acceleration signal was proceeded by an integrator (RION, UV-12, and UV-05) and converted using 12-bit $A / D$ resolution with the sampling frequency of $2 \mathrm{kHz}$, where 16,384 data points were collected in the flow time. The dynamic response frequency was analysed using the FFT analyser (ONO SOKKI, CF-5201). The angle of tip displacement $(\theta)$ for a cantilevered type is defined from Figure $1(c)$ and written as equation 3.

$\theta_{r m s}=\frac{180}{\pi} \times \frac{y_{r m s}}{L+l}$

where $I$ is plat spring length. The reduced resonance velocity $\left(V_{r}=U / f_{c} H\right)$ was ranged between 1.3 to 5.0 depending on uniform flow velocity $U$ and the characteristic frequency of the test model. The range of critical reduced velocity $\left(V_{c r}\right)$ used in this experiment is still less than the critical point of the prism as defined by Nakamura and Matsukawa [6]. The prism tip displacement was limited to around $3 \mathrm{~mm}$ to prevent plate spring failure.

\section{Results and Discussion}

\subsection{Response Amplitude}

The primary consideration focus on the prisms whose physical properties around the critical points, such as the aspect ratios (span length to width ratio, $L / H$ ) and side ratio (depth to cross-section height $D / H)$, i.e., $L \leq 5.0 H$ and $D=0.5 H$.

The detached splitter effect on the dynamic response characteristics of the rectangular prisms with different aspect ratios is shown in Figure 3. The prisms with aspect ratios $L \geq 5.0 H$ and depth $D$ $=0.2 \mathrm{H}$ exhibit similar dynamic response and onset galloping characteristics. However, the dynamic response improves at resonance velocity $V_{r}$ of $>2$. The prism with $L=2.5 \mathrm{H}$ shows distinctive dynamic responses, different from those of $L \geq 5.0 \mathrm{H}$. A slight improvement of the dynamic response occurs at high flow velocity (or $V_{r}>3$ ).

Inserting a splitter plate behind the test model with $D=0.5 \mathrm{H}$ improves the response amplitude at prisms with a span length, $L=5.0 \mathrm{H}$, and $L=2.5 \mathrm{H}$, as shown in Figure 3b. All prisms with a detached splitter plate exhibit the dynamic response improvement after the resonance velocity $V_{r}$ around 3.7. The previous studies can be considered to uncover such phenomena that the rectangular depth affects the dynamic response rectangular prism such as Kiwata et al., and Nakamura \& Tomonari [3, 26]. The vibration mechanism of the slender prisms $(D=0.2 H)$ with a sharp leading edge is solely induced by the shear layer's instability from the separation point at the leading edge and is 
independent of vortex-induced vibration. The prism depth changes the vibration mechanism in which vortex shedding downstream and reattachment are involved in the rectangular prism's dynamic response.

The previous studies $[26,27]$ presented a critical depth of the rectangular prism was 0.4, which was indicated by sudden alteration of the base pressure. Over this range, the vortex shedding effect because of the after body length is considered. For aspect ratio $L / H=10$, flow-induced vibration over the rectangular varied with various depths as shown in Kiwata et al., [3]. The waveform variation of the dynamic response for depth $D=<0.5 H$ is uniform in which the discontinuity of waveform response is not found.

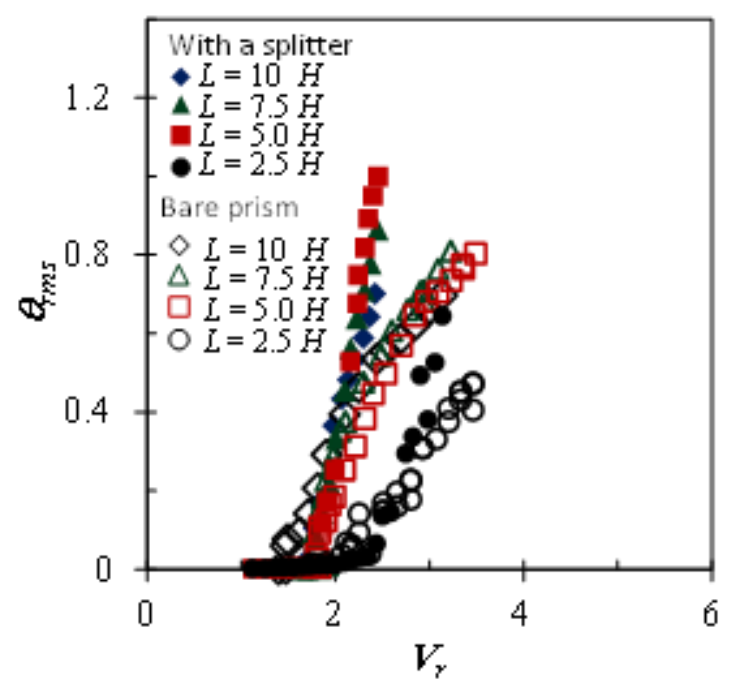

(a) $D=0.2 \mathrm{H}$

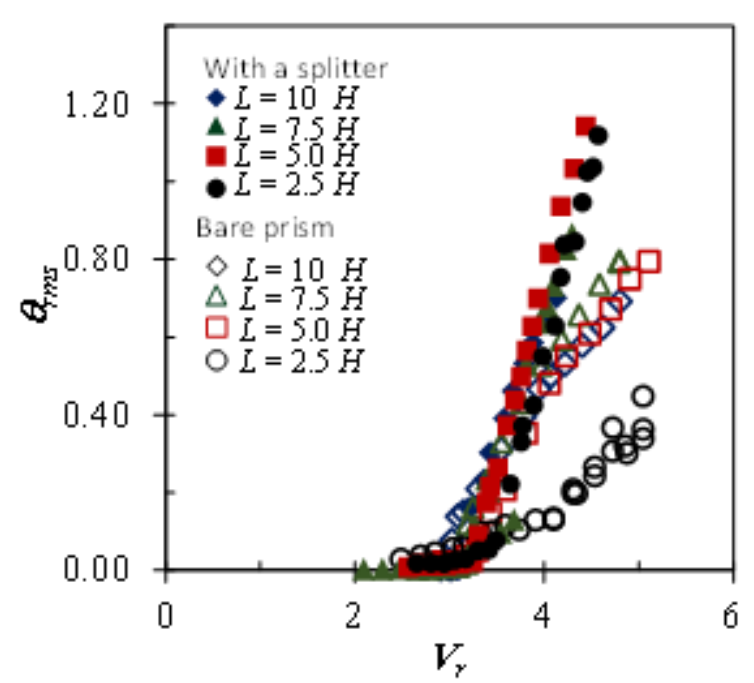

(b) $D=0.5 \mathrm{H}$

Fig. 3. Effect of a splitter plate on the response amplitude of the rectangular prism with different depths and span lengths

Figure 4 shows the FFT power spectra analysis of dynamic response at the maximum amplitude for the prism with $D=0.5 \mathrm{H}$. The velocity displacement of a prism is the part of energy transfer from the fluid to the structure during vibration motion. The frequency of displacement from time-lapses of waveform response is defined by FFT power spectra on the left side of Figure 4 . The waveform signal interference or lagging of energy transfer from the fluid to the prisms is considered at the plain prisms. It implies that the vibrated bluff body loses some energy due to the fluctuation of the hydrodynamics forces, which upticks the fluid friction. The minor disturbances of oscillation frequency adjacent to the sharp peak (Figure $4 a$ ) is found at the prism with $L / H=2.5$, and two sharp peaks (Figure $4 \mathrm{~b}$ ) on the FFT power spectra.

The highest sharp peak represents amplitude-frequency, and another one is the wake fluctuation. The intermittent waveform vanished for test models with a splitter plate and appearing only one sharp peak on the FFT power spectra analysis. Vanishing an intermittent on the second peak adjacent to the dominant peak is considered an uptick of shear layer instability and alters the critical point of the depth of the prism $[13,29]$. 


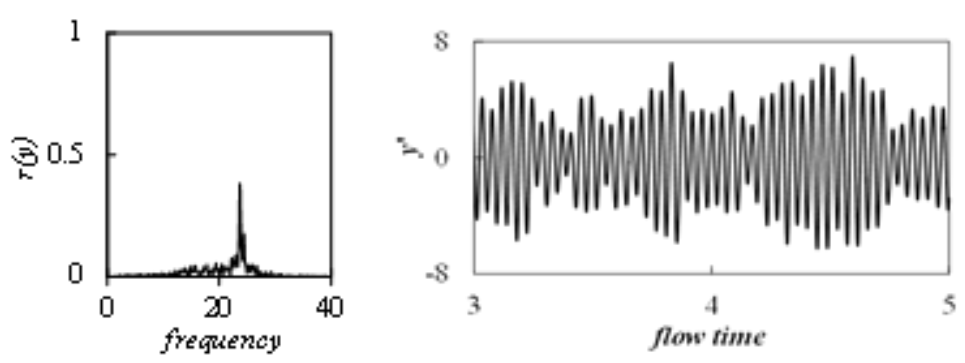

(a) $L=2.5 \mathrm{H}$ at $V_{r}=5.04$, and $U=2.66 \mathrm{~m} / \mathrm{s}$ (plain prism)
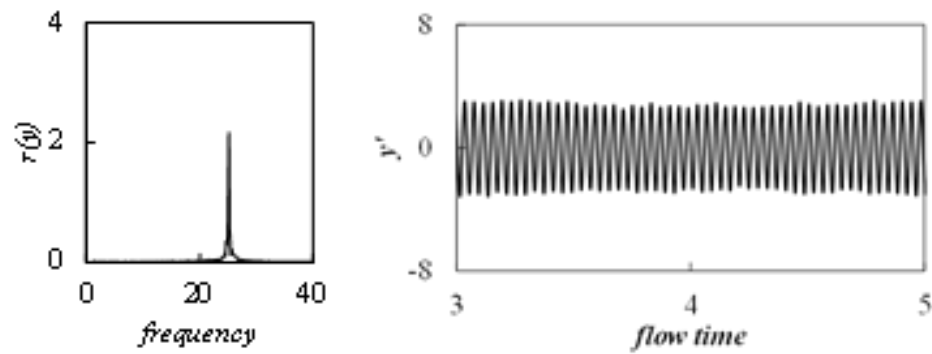

(b) $L=2.5 \mathrm{H}$ at $V_{r}=4.57$, and $U=2.41 \mathrm{~m} / \mathrm{s}$ (prism with a splitter)
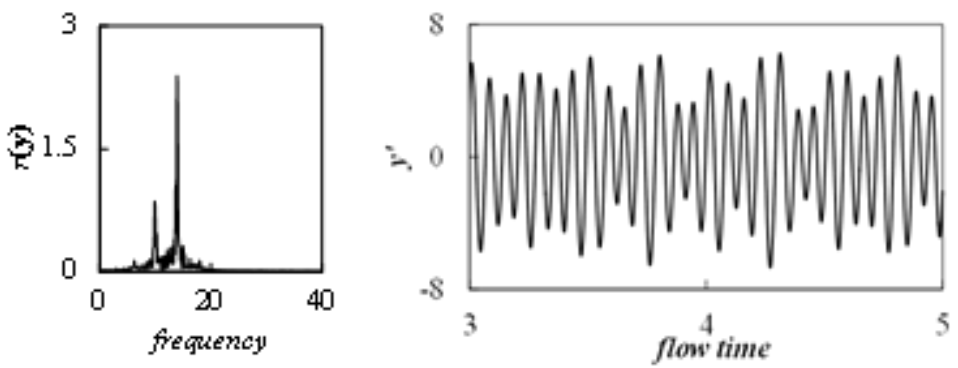

(c) $L=10 \mathrm{H}$ at $V_{r}=4.95$, and $U=1.62 \mathrm{~m} / \mathrm{s}$ (plain prism)
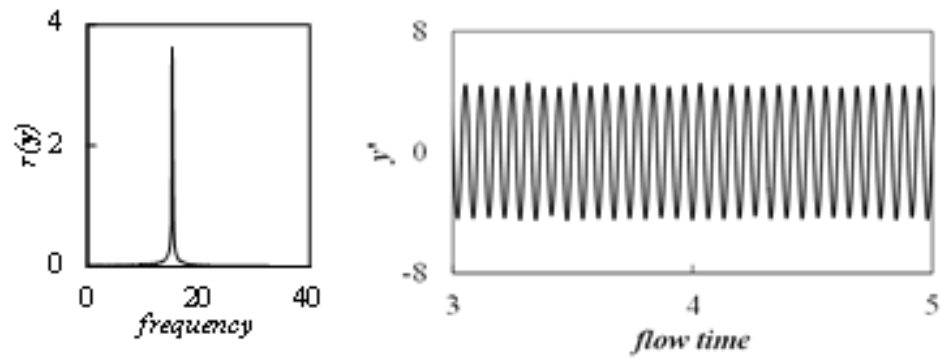

(d) $L=10 \mathrm{H}$ at $V_{r}=4.13$, and $U=1.35 \mathrm{~m} / \mathrm{s}$ (prism with a splitter)

Fig. 4. The FFT power spectra (left) and time lapses of the dynamic response of the prism with a side ratio $D / H$ of 0.5

\subsection{Peak Deviation and Increment Rate of Response Amplitude}

The effect of a detached splitter plate on the deviation of peak amplitude is shown in Figure 5. The prism below the critical point shows a noticeable stable peak, i.e., $L / H=2.5 ; D / H=0.5$. The prisms with the aspect ratios $L / H \geq 5.0$ and side ratios $D / H$ of 0.5 show a stable peak at the resonance velocity $V r \geq 3.7$ even if the peak deviation remains high at low reduced velocity. In contrast to the side ratio of 0.2 , a splitter plate's effect on the prism's peak deviation with the aspect ratios $L / H \geq 5.0$ is similar to the plain prism. It agrees with the uniformity of waveform time-lapses of the response amplitude Eliminating a discontinuity of waveform peak amplitude reduces the effect of wake fluctuation intrusion at the prism trailing edge, especially at high flow velocity. 


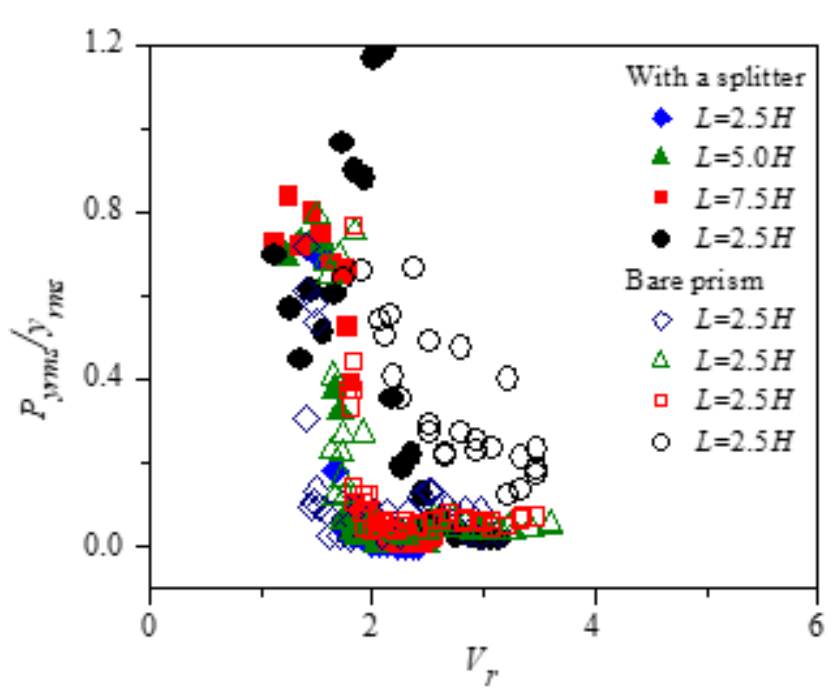

(a) $D=0.2 \mathrm{H}$

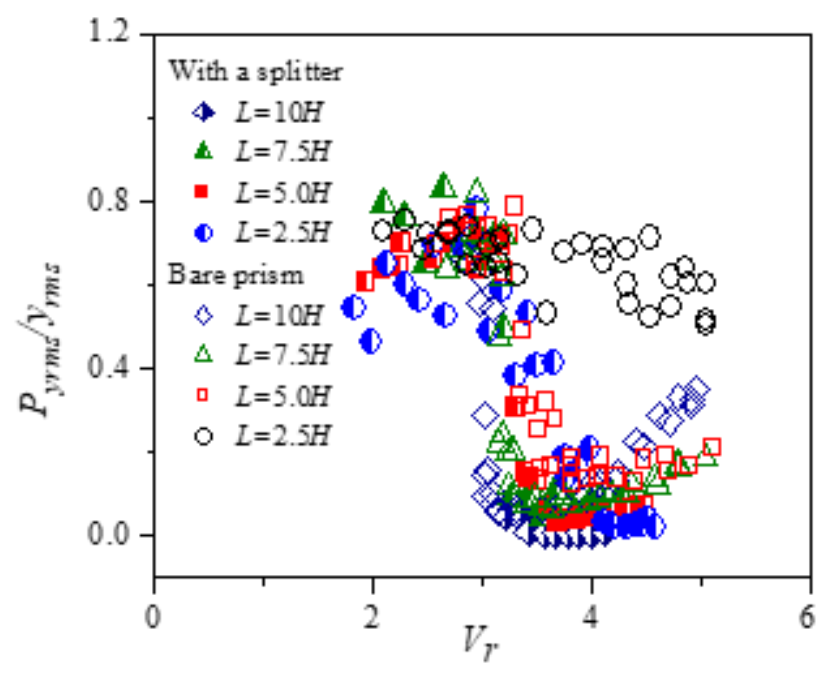

(b) $D=0.5 \mathrm{H}$

Fig. 5. Standard deviation of peak amplitude on the prisms with a splitter plate

Figure 6 presents the increment rate of response amplitude of the prisms with a fixed splitter plate. The Increment rate of response amplitude uptick by increasing the aspect ratio $\mathrm{L} / \mathrm{H}$ on both side ratios $D / H=0.2$ and 0.5 . It increases the increment rate of response amplitude for all cases. A bit of improvement is only found at the prism with $L / H 2.5$ and $D / H=0.2$. The uptick of increment rate proves that the frequency response also increases. This is an essential parameter in developing power generation from flow-induced vibration. A remarkable increase in the dynamic response increment rate is found at the slender prisms $D=0.2 H$ at aspect ratio $L / H \geq 5$ even if the displacement angle is pretty similar to plain prism at $V r<2$ (Figure 3). Preventing the vortex shedding for direct interaction upticks the galloping behaviour at a similar flow velocity with the bare prisms.

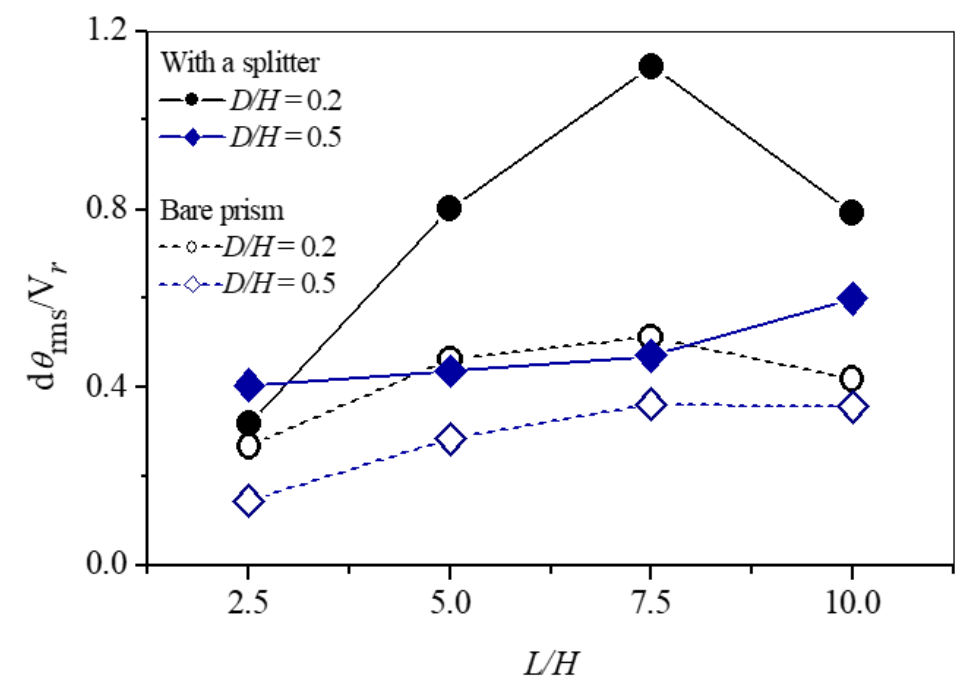

Fig. 6. Increment rate of the response amplitude

\subsection{Numerical Approach}

A brief numerical approach was carried out using a large eddy simulation (LES) turbulence model to investigate the quantities of a stationary rectangular prism with $D=0.5 \mathrm{H}$ at $\operatorname{Re}=22000$. A PISO pressure-velocity coupling method and non-slip wall condition were applied at the code. The Mesh 
model of the domain was a structural grid by considering the simulated model, such as the model's simplicity and fine grid necessity at high separation point, as shown in Figure 7 . The first cell height and growth rate of the mesh construction were determined by considering eddy motions at viscous sublayer $\left(\mathrm{y}^{+}<5\right)$. The result showed that the $\mathrm{y}^{+}$calculation is 2.1 , with a maximum value is 7.0 . It is in good agreement with the initial calculation as outlined in some references [30,31]. The residual and mass balance were monitored for convergence criterion as outlined by Oberkamf [32, 33]. The features and limitations of URANS over LES for high separation flow, such as a rectangular prism with critical side ratio $L / H<0.6$ as described by Tian et al., [34] and Yosuf et al., [35] was also considered.

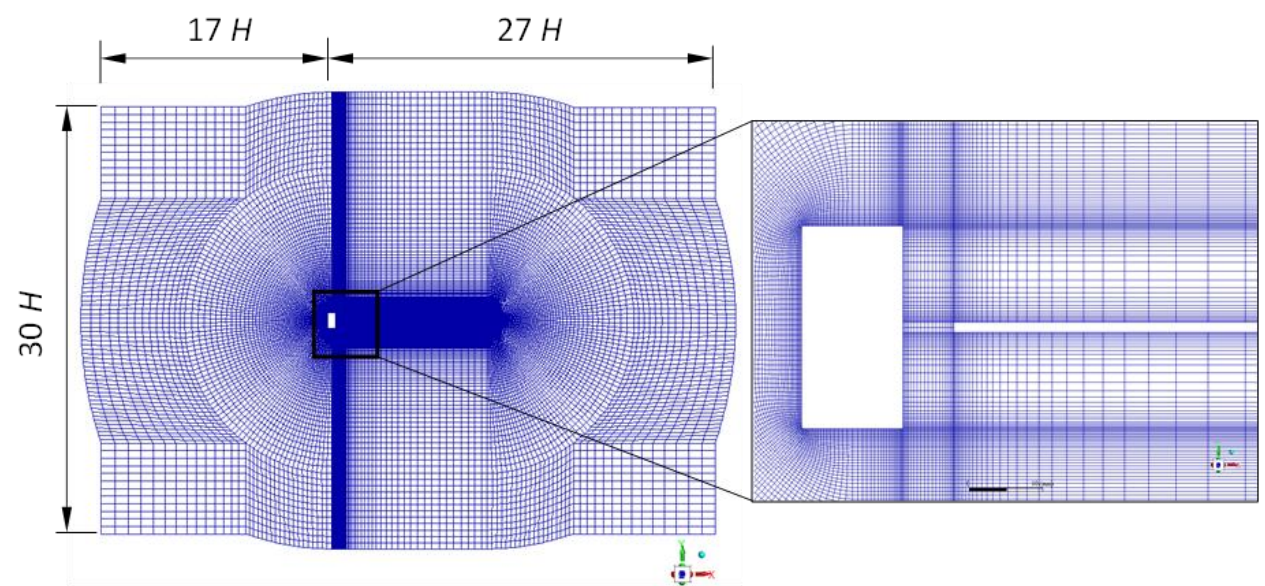

Fig. 7. Structural grid of the domain

The flow feature around a bare prism was analysed first and extended to the model with a detached splitter plate for the same approach. The flow features comparisons are shown in Table 2. The drag and base pressure coefficient are sensitive to side ratio $(D / H)$ variation, where the depth $D$ $=\sim 0.6 \mathrm{H}$ is the critical depth. The Strouhal number of a rectangular prism with $D / H \leq 1.0$ is less sensitive than hydrodynamics forces. As shown in Table 2, the drag and base pressure coefficients of a plain prism agree with other numerical calculations and are slightly different from the experimental investigations. The Strohual frequency is also reasonable agreement for both numeric and experimental investigations.

\section{Table 2}

The flow features of the quantities around a bare prism and detached splitter plate

\begin{tabular}{|c|c|c|c|c|c|c|}
\hline \multicolumn{7}{|l|}{ Bare Prism } \\
\hline & $D / H$ (Square) & $\mathrm{CD}$ & $-\mathrm{Cpb}$ & St & $\operatorname{Re}$ & Remark \\
\hline Tamura [36] & $0.5(-)$ & 2.82 & - & 0.13 & $\times 10^{4}$ & 3D Numeric \\
\hline Mizukami [25] & $0.5(1.0)$ & $2.84(2.15)$ & $2.34(1.43)$ & $0.13(0.12)$ & $2.2 \times 10^{4}$ & LES 3D \\
\hline YU [37] & $0.5(1.0)$ & $3.03(2.14)$ & $2.37(1.56)$ & $0.17(0.14)$ & $10^{4}$ & LES 3D \\
\hline Sohankar [38] & $0.4(1.0)$ & $2.49(2.20)$ & $1.75(1.57)$ & $0.15(0.12)$ & $10^{4}$ & LES 3D \\
\hline Knisley [39] & $0.5(1.0)$ & $2.33(2.03-2.20)$ & & $0.14(0.13)$ & $2.2 \times 10^{4}$ & Exp. \\
\hline Bearman [40] & $0.5(1.0)$ & $2.40(2.19)$ & $1,62(1.4)$ & $0.13(0.12)$ & $2-7 \times 10^{4}$ & Exp. \\
\hline Present & 0.5 & $2.80(-)$ & $2.40(-)$ & $0.14(-)$ & $2.2 \times 10^{4}$ & LES 3D \\
\hline \multicolumn{7}{|c|}{ With a splitter plate } \\
\hline & $D / H$ (Square) & $C D$ & $-\mathrm{Cpb}$ & St & $\operatorname{Re}$ & Remark \\
\hline Bearman [40] & $0.5(1.0)$ & $1.37(1.34)$ & 0.59 & & $2-7 \times 10^{4}$ & Exp. \\
\hline Park [42] & $-(1.0)$ & 1.47 & & $(0.053)$ & & Numeric \\
\hline Ali [43] & $-(1.0)$ & $-(1.26)$ & & $-(0.015)$ & $1.5 \times 10^{2}$ & Numeric \\
\hline Present & 0.5 & 1.38 & 0.58 & 0.053 & $2.2 \times 10^{4}$ & Numeric \\
\hline
\end{tabular}


In the case of a prism with a splitter plate, the calculation result on drag and base pressure coefficient match Bearman's work [39]. The length of the splitter plate is long enough to counter vortex shedding downstream and reattach to the splitter plate's body, as suggested by Bearman [40]. However, they did not show the effect of the gap between the prism and splitter. Ali et al., [41] have shown a critical gap of $\sim 2.5$ for a square cylinder with a detached splitter plate. This study considers the gap ratio is 0.5 . We consider that the flow pattern of a square cylinder is different from a rectangular prism with depth below critical depth. It is a curiosity for future work.

Some comparisons are shown to understand the detached splitter plate's effect on the dynamic response of elastically mounted blunt prisms. Figure 8 compares the local vortex region in the flow field around a bare and rectangular with a detached splitter plate. In a bare prism, the vortex region at the after body exhibit unsymmetrical swirling vortices, which is different from its counterpart. Consequently, the alteration of flow features such as the base suction, hydrodynamic forces, and Karman vortices street is distinctly different from the prism with a detached splitter plate.

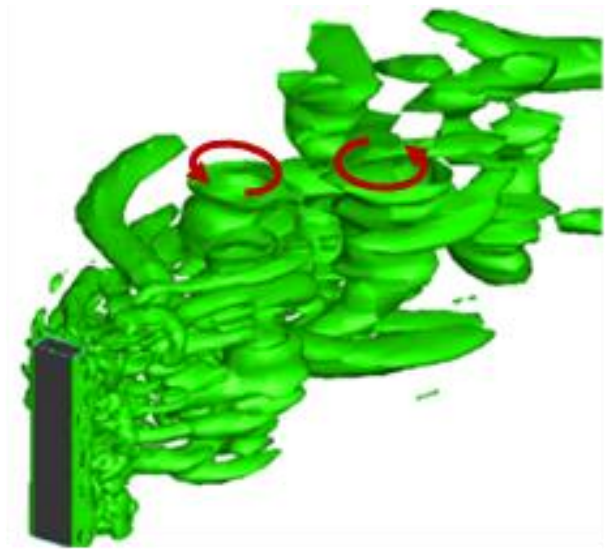

(a)

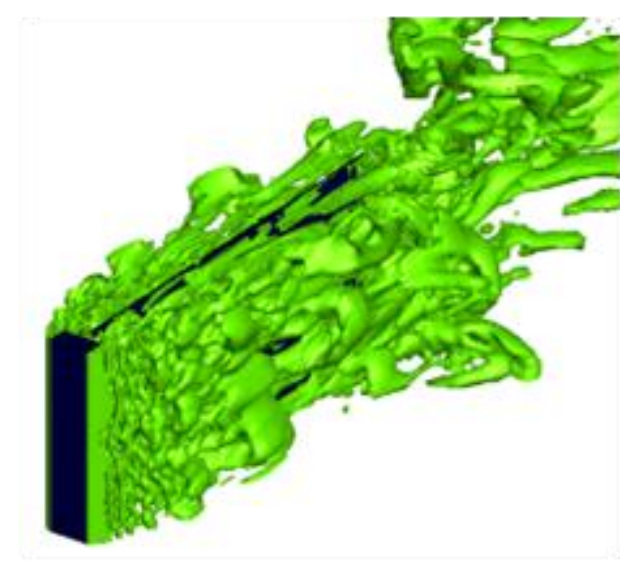

(b)

Fig. 8. Vortex region around after body of rectangular prism on two different conditions. (a) Without a splitter plate (b) With a detached splitter plate

Figure 9 shows the effect of a detached splitter plate on wake development at the after body. The splitter plate with length $L=10 \mathrm{H}$ effectively prevent shear layer entrainment in the after body resulting in a symmetrical pattern of vortices. The presence of a splitter plate creates cavities downstream, and it makes recovery of the base pressure. Reattachment of the separated shear layer on the fixed splitter plate does not affect the prism's dynamic response because it is detached from the bluff body. Potent vortex entrainment is found at the plain prism.

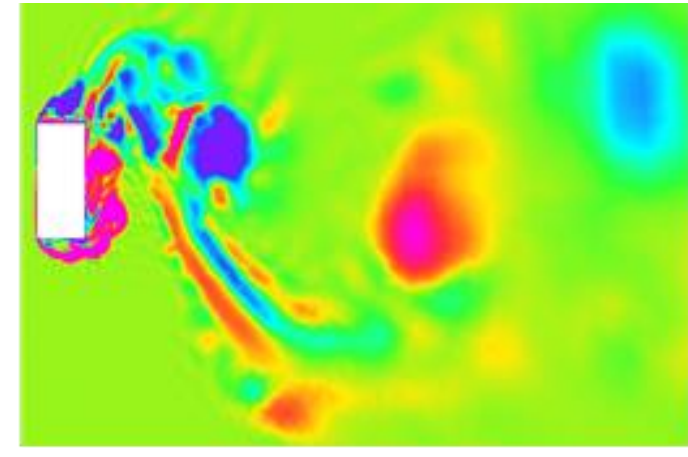

(a) Prism without a splitter plate

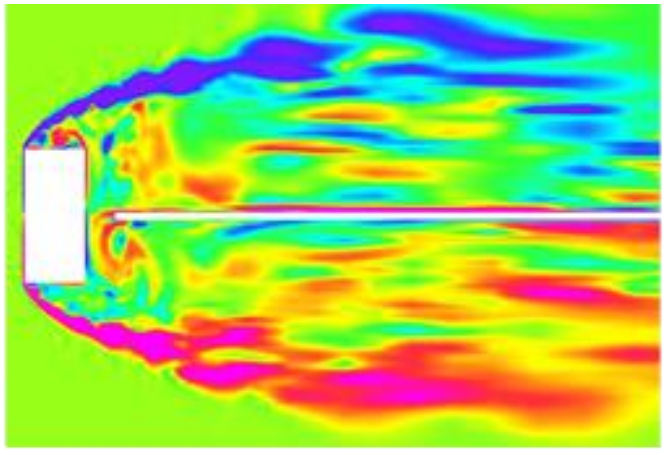

(b) Prism with a splitter plate

Fig. 9. The middle plane of vorticity around the prisms at $t=4$ second 
In contrast, the shear layer interaction is diminished in the prism with a splitter plate. The splitter plate length $L=10 \mathrm{H}$ is also sufficient to weaken vortex shedding streamwise. The evolution of separated shear layer characteristics behind the prims changed base pressure and hydrodynamic forces such as lift and drag force, as shown in Figures 10 and 11.

(a)

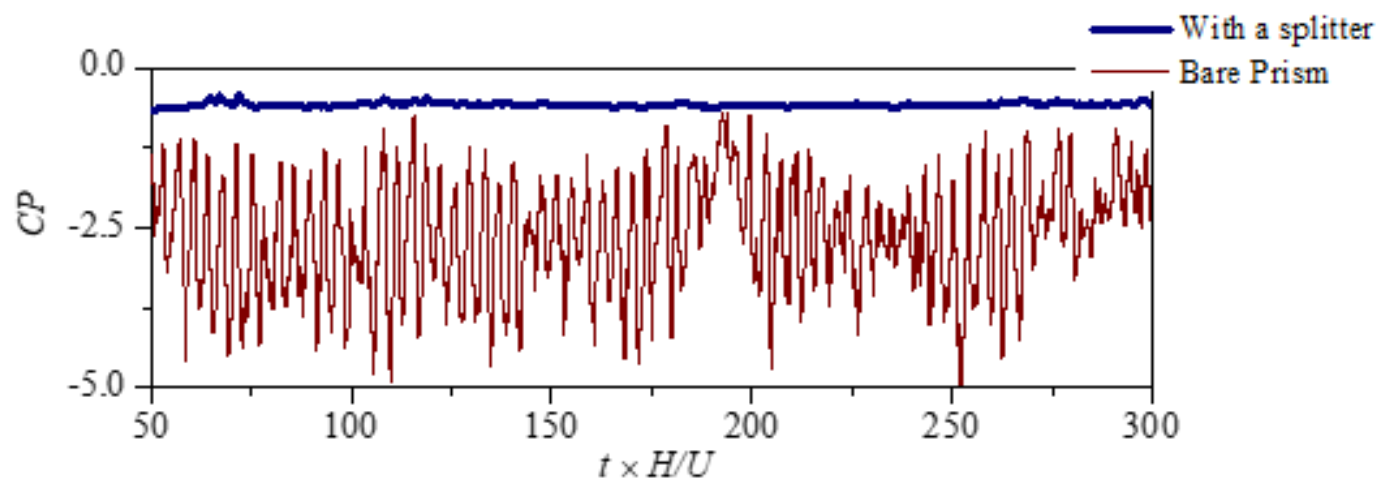

(b)

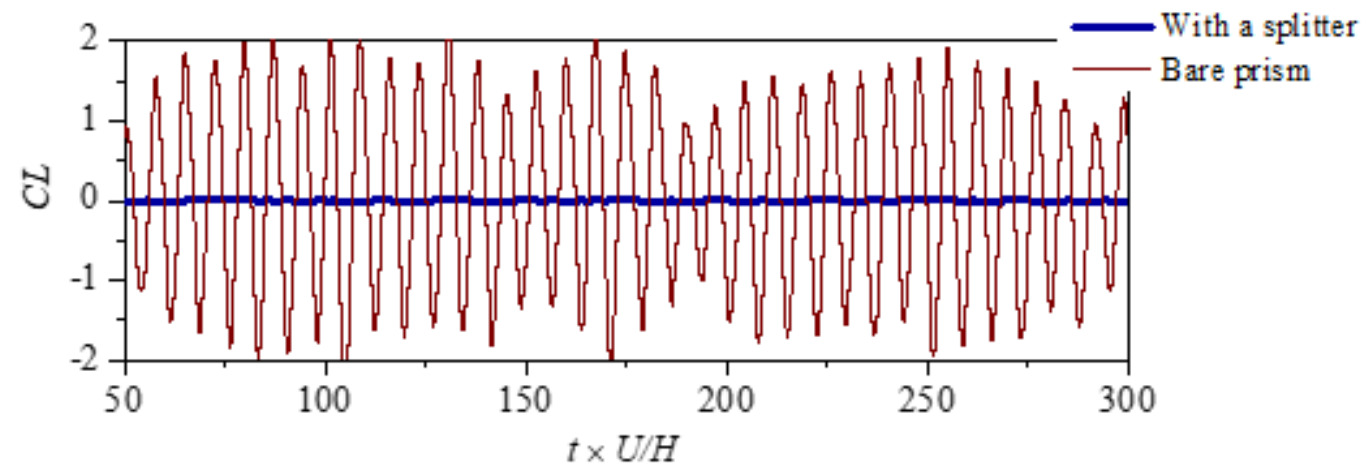

(c)

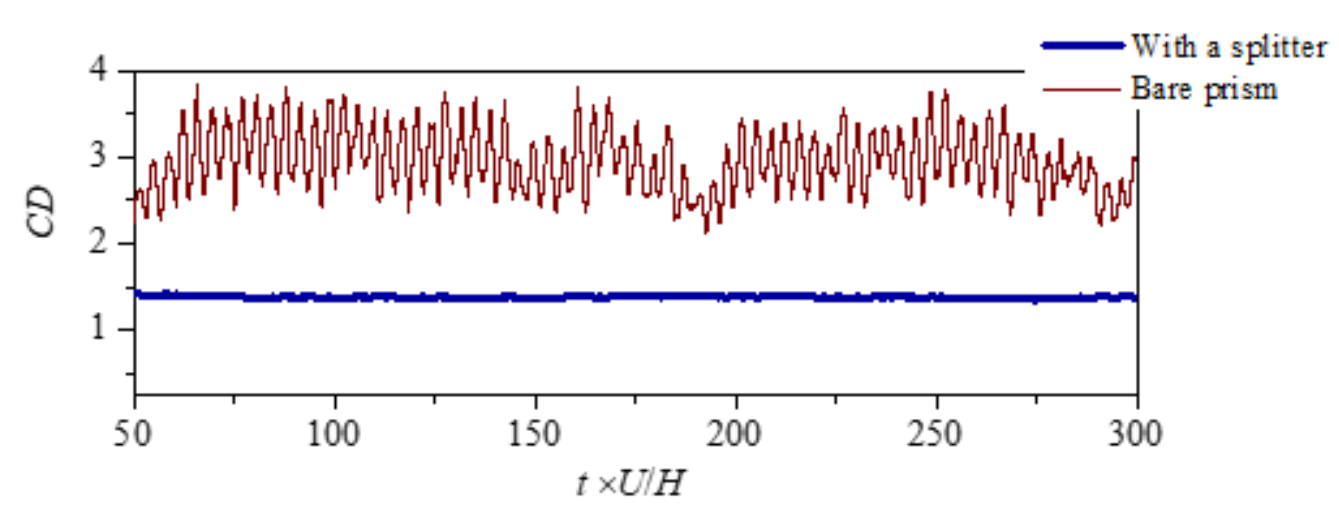

Fig. 10. Characteristics of the flow features around the prism. (a) Pressure coefficient, (b) Lift Coefficient, (c) Drag Coefficient

The fluctuation of hydrodynamic forces and pressure is suppressed during the flowtime. Figure 11 exhibits the effect of a detached splitter plate on base suction. It shows that reducing flow entrainment at the base improves the pressure coefficient $(C P)$ at the prism with a fixed splitter plate. Moreover, a non-dimensional wake shedding frequency is also shown in Figure 12. Prism with a detached splitter plate presents a weak fluctuation vortex shedding, while a bare prism shows a sharp peak of shedding fluctuation. It seems that vortex shedding in the after body takes an essential role for some quantities, such as pressure and hydrodynamic force. They are related to the dynamic response of the elastically mounted structure. 


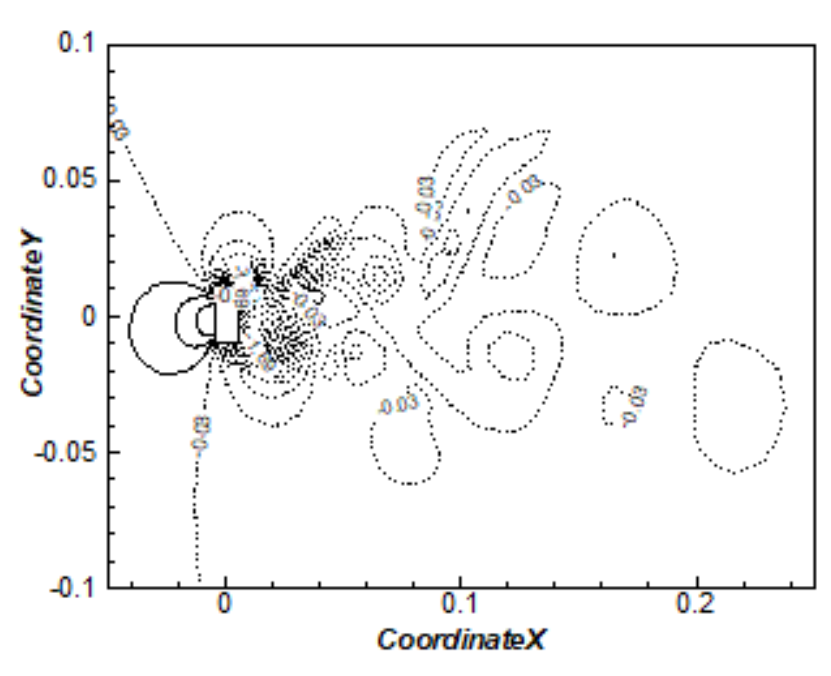

(a) Prism without a splitter plate

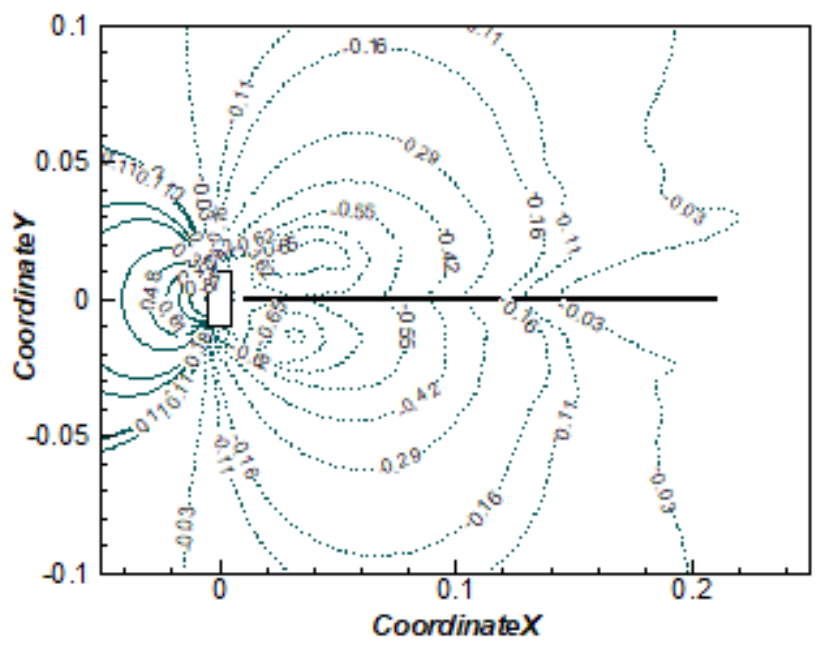

(b) Prism with a splitter plate

Fig. 11. Pressure contours

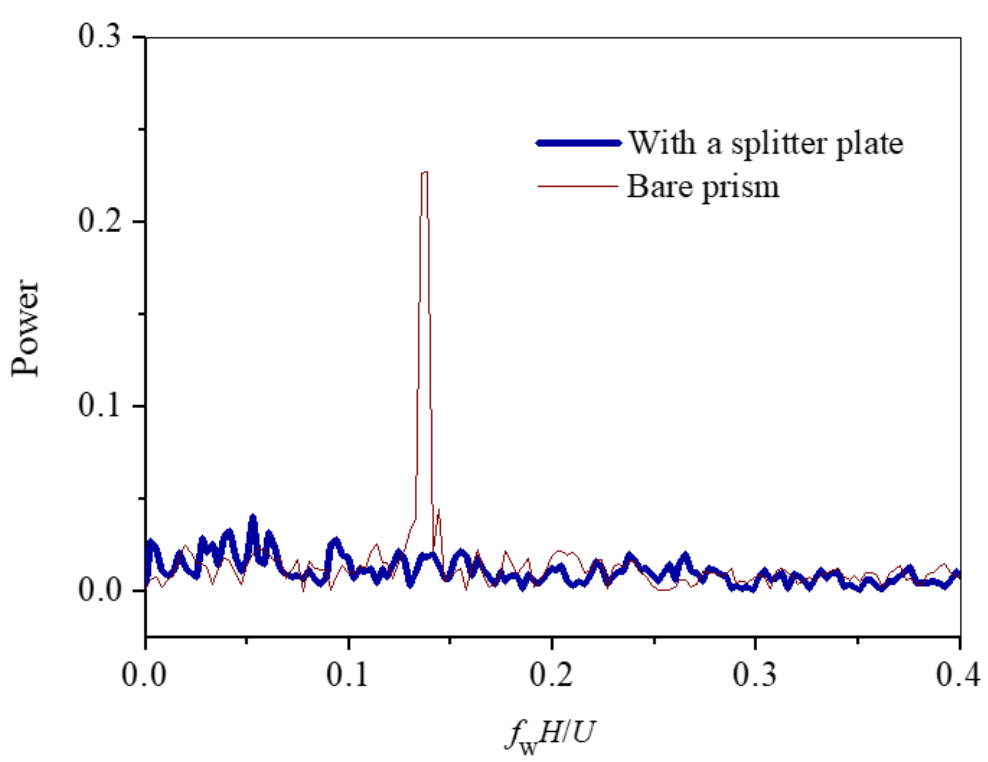

Fig. 12. The non-dimensional vortex shedding frequency

\section{Conclusions}

The experiment of flow-induced vibration of cantilevered rectangular prisms with a detached splitter plate in a water tunnel had been accomplished with the following conclusions:

i. Mounting a splitter plate at prism trailing edge improve response amplitude for both cross-section models by modifying the vortex dynamic behind the prisms instead of allowing them to interact directly.

ii. Mounting a splitter plate in rectangular prisms with aspect ratios $\mathrm{L} / \mathrm{H} \leq 5.0$ and a side ratio of 0.2 does not affect response amplitude. It diminishes interference of peak amplitude on the prism with $\mathrm{D} / \mathrm{H}=0.5$ and uptick the increment rate of response amplitude.

iii. The stable peak amplitude is achieved by inserting a splitter plate behind the prism with a side ratio of 0.5 for all aspect ratios $\mathrm{L} / \mathrm{H}$. 
iv. A splitter plate stabilises vortex shedding at trailing edge, increases base pressure, reduces drag force and Strouhal frequency.

v. Inserting a splitter plate is a prospective method to enhance power generation performance from flow-induced vibration

\section{References}

[1] Naudascher, Eduard, and Donald Rockwell. Flow-induced vibrations: an engineering guide. Routledge, 2017.

[2] Okajima, Atsushi. "Strouhal numbers of rectangular cylinders." Journal of Fluid mechanics 123 (1982): 379-398. https://doi.org/10.1017/S0022112082003115

[3] Kiwata, Takahiro, Makoto Yamaguchi, Takaaki Kono, and Toshiyuki Ueno. "Water tunnel experiments on transverse-galloping of cantilevered rectangular and D-section prisms." Journal of Fluid Science and Technology 9 , no. 3 (2014): JFST0056-JFST0056. https://doi.org/10.1299/jfst.2014jfst0056

[4] Nakamura, Y., and K. Hirata. "Pressure fluctuations on oscillating rectangular cylinders with the long side normal to the flow." Journal of fluids \& structures 5, no. 2 (1991): 165-183. https://doi.org/10.1016/0889-9746(91)90460-7

[5] Skop, R. A., and O. M. Griffin. "On a theory for the vortex-excited oscillations of flexible cylindrical structures." Journal of Sound and Vibration 41, no. 3 (1975): 263-274. https://doi.org/10.1016/S0022460X(75)80173-8

[6] Nakamura, Yasuharu, and Tohru Matsukawa. "Vortex excitation of rectangular cylinders with a long side normal to the flow." Journal of fluid mechanics 180 (1987): 171-191. https://doi.org/10.1017/S0022112087001770.

[7] Palau-Salvador, Guillermo, Thorsten Stoesser, Jochen Fröhlich, Michael Kappler, and Wolfgang Rodi. "Large eddy simulations and experiments of flow around finite-height cylinders." Flow, turbulence and combustion 84, no. 2 (2010): 239-275. https://doi.org/10.1007/s10494-009-9232-0.

[8] Sumner, D., N. Rostamy, D. J. Bergstrom, and J. D. Bugg. "Influence of aspect ratio on the mean flow field of a surface-mounted finite-height square prism." International Journal of Heat and Fluid Flow 65 (2017): 1-20. https://doi.org/10.1016/J.IJHEATFLUIDFLOW.2017.02.004.

[9] Wang, Hanfeng, Xiaoyan Zhao, Xuhui He, and Yu Zhou. "Effects of oncoming flow conditions on the aerodynamic forces on a cantilevered square cylinder." Journal of Fluids and Structures 75 (2017): 140-157. https://doi.org/10.1016/J.JFLUIDSTRUCTS.2017.09.004.

[10] Kiwata, Takahiro, Takaaki Kono, and Toshiyuki Ueno. "Effects of Span Length and Additional Structure on FlowInduced Transverse Vibration Characteristic of a Cantilevered Rectangular Prism." Journal of Flow Control, Measurement \& Visualization 8, no. 3 (2020): 102-120. https://doi.org/10.4236/ifcmv.2020.83006.

[11] Gonçalves, R. T., G. R. Franzini, Guilherme F. Rosetti, J. R. Meneghini, and A. L. C. Fujarra. "Flow around circular cylinders with very low aspect ratio." Journal of Fluids and Structures 54 (2015): 122-141. https://doi.org/10.1016/J.JFLUIDSTRUCTS.2014.11.003.

[12] Zdravkovich, M. M. "Review and classification of various aerodynamic and hydrodynamic means for suppressing vortex shedding." Journal of Wind Engineering and Industrial Aerodynamics 7, no. 2 (1981): 145-189. https://doi.org/10.1016/0167-6105(81)90036-2.

[13] Nakamura, Y., K. Hirata, and T. Urabe. "Galloping of rectangular cylinders inthe presence of a splitter plate." Journal of fluids and structures 5, no. 5 (1991): 521-549. https://doi.org/10.1016/S0889-9746(05)80004-0.

[14] Qiu, Y., Y. Sun, Y. Wu, and Y. Tamura. "Effects of splitter plates and Reynolds number on the aerodynamic loads acting on a circular cylinder." Journal of Wind Engineering and Industrial Aerodynamics 127 (2014): 40-50. https://doi.org/10.1016/i.jweia.2014.02.003.

[15] Ogunremi, A. R., and D. Sumner. "The effect of a splitter plate on the flow around a finite prism." Journal of Fluids and Structures 59 (2015): 1-21. https://doi.org/10.1016/i.jluidstructs.2015.09.001.

[16] Chauhan, Manish Kumar, Sushanta Dutta, Bhupendra Singh More, and Bhupendra Kumar Gandhi. "Experimental investigation of flow over a square cylinder with an attached splitter plate at intermediate Reynolds number." Journal of Fluids and Structures 76 (2018): 319-335. https://doi.org/10.1016/i.jfluidstructs.2017.10.012.

[17] Aabid, Abdul, Azmil Afifi, Fharuk Ahmed Ghasi Mehaboob Ali, and Mohammad Nishar Akhtar Sher Afghan Khan. "CFD Analysis of Splitter Plate on Bluff Body." CFD Letters 11, no. 11 (2019): 25-38.

[18] Sahu, Tulsi Ram, Mohd Furquan, and Sanjay Mittal. "Numerical study of flow-induced vibration of a circular cylinder with attached flexible splitter plate at low." Journal of Fluid Mechanics 880 (2019): 551-593. https://doi.org/10.1017/jfm.2019.699.

[19] Xu Sun , C. Steve Suh , Ze-Hua Ye, and Bo Yu. "Flow Control over a Square Cylinder Using Attached Rigid and Flexible Splitter Plate at Intermediate Flow Regime." Physics of Fluids 32, no. 1: 014104. https://doi.org/10.1063/1.5127905.

[20] Sharma, K. R., and S. Dutta. "Flow control over a square cylinder using attached rigid and flexible splitter plate at 
intermediate flow regime." Physics of Fluids 32, no. 1 (2020): 014104. https://doi.org/10.1063/1.5127905.

[21] Assi, Gustavo R.S., and Peter W. Bearman. "Transverse Galloping of Circular Cylinders Fitted with Solid and Slotted Splitter Plates." Journal of Fluids and Structures $54 \quad$ (2015): $263-280$. https://doi.org/10.1016/j.jfluidstructs.2014.11.005.

[22] Hwang, Jong-yeon, and Kyung-soo Yang. "Drag Reduction on a Circular Cylinder Using Dual Detached Splitter Plates." Journal of Wind Engineering and Industrial Aerodynamics 95 (2007): 551-564. https://doi.org/10.1016/i.jweia.2006.11.003.

[23] Barman, Bhanuman, and Somnath Bhattacharyya. "Control of Vortex Shedding and Drag Reduction through Dual Splitter Plates Attached to a Square Cylinder." Journal of Marine Science and Application, (2015) https://doi.org/10.1007/s11804-015-1299-5.

[24] Dash, Sunil Manohar, Michael S. Triantafyllou, and Pablo Valdivia Y. Alvarado. "A numerical study on the enhanced drag reduction and wake regime control of a square cylinder using dual splitter plates." Computers \& Fluids 199 (2020): 104421. https://doi.org/10.1016/i.compfluid.2019.104421.

[25] Mizukami, Shunichi. 2017. "Study on the Flow around the Elastic Supported Prism and the Vibration Dynamics of the Flow (in Japanese)." Master Thesis, Graduate School of Natural Science and Technology, Kanazawa University.

[26] Nakamura, Y., and Y. Tomonari. "Galloping of rectangular prisms in a smooth and in a turbulent flow." Journal of Sound and Vibration 52, no. 2 (1977): 233-241. https://doi.org/10.1016/0022-460X(77)90642-3.

[27] Nakamura, Yasuharu, and Katsuya Hirata. "Critical geometry of oscillating bluff bodies." Journal of Fluid Mechanics 208 (1989): 375-393. https://doi.org/10.1017/S0022112089002879.

[28] Pattenden, R. J., S. R. Turnock, and Xin Zhang. "Measurements of the flow over a low-aspect-ratio cylinder mounted on a ground plane." Experiments in Fluids 39, no. 1 (2005): 10-21. https://doi.org/10.1007/s00348-005-0949-9.

[29] Ohya, Yuji. "Note on a discontinuous change in wake pattern for a rectangular cylinder." Journal of fluids and structures 8, no. 3 (1994): 325-330. https://doi.org/10.1006/jfls.1994.1015

[30] Kajishima, Takeo, and Kunihiko Taira. "Computational Fluid Dynamics." In Springer Tracts in Mechanical Engineering, 87-204, 2018. https://doi.org/10.1007/978-3-319-76234-0 3.

[31] H K Versteeg and W Malalasekera. "Introduction to Computational Fluid Dynamics." Introductory Fluid Mechanics, 2012. https://doi.org/10.1017/cbo9780511761348.011.

[32] Oberkampf, William L., and Timothy G. Trucano. Verification and Validation in Computational Fluid Dynamics. Progress in Aerospace Sciences. Vol. 38, 2002. https://doi.org/10.1016/S0376-0421(02)00005-2.

[33] Oberkampf, William L., and Matthew F. Barone. "Measures of Agreement between Computation and Experiment: Validation Metrics." Journal of Computational Physics 217, no. 1 (2006): 5-36. https://doi.org/10.1016/j.jcp.2006.03.037.

[34] Tian, Xinliang, Muk Chen Ong, Jianmin Yang, and Dag Myrhaug. "Unsteady RANS Simulations of Flow around Rectangular Cylinders with Different Aspect Ratios." Ocean Engineering 58 (2013): 208-216. https://doi.org/10.1016/j.oceaneng.2012.10.013

[35] Yusuf, Siti Nurul Akmal, Yutaka Asako, Nor Azwadi Che Sidik, Saiful Bahri Mohamed, and Wan Mohd Arif Aziz Japar. "A Short Review on RANS Turbulence Models." CFD Letters 12, no. 11 (2020): 83-96. https://doi.org/10.37934/cfdl.12.11.8396

[36] Tamura, T., and Y. Itoh. "Three-dimensional vortical flows around a bluff cylinder in unstable oscillations." Journal of wind engineering and industrial aerodynamics 67 (1997): 141-154. https://doi.org/10.1016/S01676105(97)00069-X

[37] Yu, Dahai, Kyle Butler, Ahsan Kareem, James Glimm, and Jiangang Sun. "Simulation of the Influence of Aspect Ratio on the Aerodynamics of Rectangular Prisms." Journal of Engineering Mechanics 139, no. 4 (2013): $429-438$. https://doi.org/10.1061/(ASCE)EM.1943-7889.0000494.

[38] Sohankar, A. "Large Eddy Simulation of Flow Past Rectangular-Section Cylinders: Side Ratio Effects." Journal of Wind Engineering and Industrial Aerodynamics 96, no. 5 (2008): 640-655. https://doi.org/10.1016/i.jweia.2008.02.009.

[39] Knisely, C. W. "Strouhal Numbers of Rectangular Cylinders at Incidence: A Review and New Data." Journal of Fluids and Structures 4, no. 4 (1990): 371-393. https://doi.org/10.1016/0889-9746(90)90137-T.

[40] Bearman, P W, and D M Trueman. "An Investigation of the Flow around Rectangular Cylinders." Aeronautical Quarterly 23, no. 3 (1972): 229-237. https://doi.org/10.1017/S0001925900006119

[41] Ali, Mohamed Sukri Mat, Con J. Doolan, and Vincent Wheatley. "Low Reynolds Number Flow over a Square Cylinder with a Detached Flat Plate." International Journal of Heat and Fluid Flow 36 (2012): $133-141$. https://doi.org/10.1016/j.ijheatfluidflow.2012.03.011.

[42] Park, Woe Chul. "Numerical Investigation of Wake Flow Control by a Splitter Plate." KSME International Journal 12, no. 1 (1998): 123-131. https://doi.org/10.1007/BF02946540.

[43] Mat Ali, Mohamed Sukri, Con J. Doolan, and Vincent Wheatley. "Low Reynolds Number Flow over a Square Cylinder with a Splitter Plate." Physics of Fluids 23, no. 3 (2011). https://doi.org/10.1063/1.3563619. 